\title{
Learning Curve of a Surgical Educator: Resident Cataract Surgery Outcomes under the Supervision of One Attending Surgeon over 5 Years
}

\author{
Sidra Zafar, MD ${ }^{1}$ Ariel Chen, MD ${ }^{1}$ Seung Yeon Seo, BS ${ }^{1}$ Xinyi Chen, BS ${ }^{1}$ jing Tian, MS² \\ Divya Srikumaran, MD $^{1}$ Shameema Sikder, MD $^{1}$ Fasika A. Woreta, MD, MPH ${ }^{1}$

\footnotetext{
1 Department of Ophthalmology, Wilmer Eye Institute, Johns Hopkins Hospital, Baltimore, Maryland

2 Johns Hopkins University School of Medicine and Bloomberg School of Public Health, Johns Hopkins University, Baltimore, Maryland
} \\ Address for correspondence Fasika A. Woreta, MD, MPH, Wilmer Eye \\ Institute, 600 North Wolfe St., Baltimore, MD 21287 \\ (e-mail: fworeta1@jhmi.edu). \\ J Acad Ophthalmol 2020;12:e15-e19.
}

\begin{abstract}
Background The main purpose of this article is to determine the trend of intraoperative complications of resident-performed cataract surgery under the supervision of one attending surgeon during the initial 5 years of surgical teaching.

Methods A retrospective chart review was conducted at the Wilmer Eye Institute for all postgraduate year 4 resident-performed cataract surgeries between July 2013 and June 2018 that were supervised by one attending surgeon who was directly out of fellowship training. Recorded data included patient demographics, presence of preoperative risk factors (small pupil, dense cataract, history of ocular trauma, pseudoexfoliation syndrome, postvitrectomy, pre-existing zonular weakness, and high myopia), and intraoperative complications (posterior capsule tear resulting in vitreous loss and zonular dehiscence resulting in vitreous loss and/or loss of sulcus support). A generalized estimating equation model was constructed to determine factors associated with intraoperative complications.

Results A total of 1,253 resident cataract surgeries involving 988 patients were included. The overall complication rate was $3.6 \%(n=45 / 1253)$, with a decreasing trend observed in the rate of intraoperative complications across the study years. Multivariate analysis found that complications during cataract surgery were more likely to occur in earlier years of the supervising surgeon's career $(p=0.01)$, at the beginning of the academic year $(p=0.03)$, in eyes with preoperative risk factors $(p<0.001)$, black

Keywords

- learning curve

- novice surgeon

- surgical educator

- complications patients $(p=0.02)$, and left eyes $(p=0.02)$.

Conclusion A learning curve exists for novice attending surgeons during their initial years of resident cataract surgery supervision. Residency programs should consider resources to educate novice surgical educators, especially on how to effectively supervise resident-performed complex cases.
\end{abstract}

The learning curve for resident-performed phacoemulsification has been well-described in the literature, with multiple studies reporting decreasing intraoperative complication rates as resident surgical volume increases. ${ }^{1,2}$ While surgical expe- rience remains important, several additional factors are also known to influence the operative learning curve including the presence of a structured training curriculum for learners ${ }^{3,4}$ and supervision. ${ }^{5}$ received

August 27, 2019

accepted after revision

December 1, 2019
DOI https://doi.org/

$10.1055 / \mathrm{s}-0039-3402771$.

ISSN 2475-4757.
Copyright $\odot 2020$ by Thieme Medical Publishers, Inc., 333 Seventh Avenue, New York, NY 10001, USA. Tel: +1(212) 584-4662.
License terms

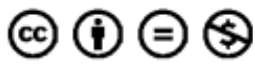


Even with the implementation of a structured curriculum, a substantial amount of resident teaching occurs in the operating room. Supervision in the operating room often enables attending surgeons to identify and provide detailed feedback to residents in areas needing improvement. Attending guidance also prevents potentially dangerous or inappropriate movements and allows for a more experienced surgeon to take over the surgery when necessary. Moreover, the teaching provided by the supervising attending surgeon often plays an important role in the resident's entire approach to surgery. Operating room supervision is therefore not only important in shaping the resident's learning experience but is also crucial to patient safety.

Recent studies suggest that operative learning curves for certain procedures, including phacoemulsification, exhibit ongoing improvement over the course of a surgeon's career. ${ }^{6,7}$ This may be particularly relevant to surgeons who have recently graduated, as lack of experience may be associated with higher complication rates early on. ${ }^{8}$ By extension, the relative inexperience of the newly independent surgeon may potentially be associated with deficiencies in resident supervision and teaching. A 2015 study comparing resident cataract surgery outcomes supervised by a novice versus an experienced attending surgeon found that resident cases supervised by the novice surgeon had significantly higher rates of vitreous loss and anterior capsule tears. ${ }^{\text {? }}$

While many studies have observed the complex relationships between trainee experience and complications, ${ }^{1,9}$ there is a limited understanding of how attending experience may impact resident surgical success. Thus, the purpose of our study is to assess intraoperative complication rates of resident-performed phacoemulsification supervised by a single attending over a continuous period of 5 years. To our knowledge, this is the first study to analyze the longterm trend of an attending directly out of training supervising resident operative cases.

\section{Methods}

\section{Case Selection}

This was a retrospective study conducted at the Wilmer Eye Institute, Johns Hopkins Hospital. A retrospective review was performed for all patients who underwent resident-performed cataract surgeries supervised by a single attending at our institute between July 2013 and June 2018. The designated primary surgeon for each case was a postgraduate year 4 (PGY4) ophthalmology resident and all cases during the 5 -year study period were supervised by the same attending surgeon who had recently completed a cornea fellowship. The study adhered to the Tenants of the Declaration of Helsinki and was conducted after approval from the Institutional Review Board of the Johns Hopkins Hospital. Since this was a retrospective study and all patient data was deidentified, individual patient consent was not required.

\section{Data Collection}

Recorded data included information on patient demographics (age, sex, race/ethnicity), academic year, presence of preoper- ative risk factors (small pupil defined as pupil dilation of $<6 \mathrm{~mm}$ or $\alpha$-adrenergic use, white or brunescent cataract graded as $4+$ or more, history of ocular trauma, pseudoexfoliation syndrome, prior history of vitrectomy, pre-existing zonular weakness, and high myopia defined as greater than -6 diopters), laterality of the eye, intraoperative use of trypan blue or iris expansion devices, nuclear disassembly technique used (divide and conquer, stop and chop, pop and chop, primary chop, irrigation and aspiration, or small incision cataract surgery) and intraoperative complications. Cases were classified as complex if one or greater preoperative risk factors were present. The occurrence of major intraoperative complications, defined as an anterior or posterior capsule tear resulting in vitreous loss or zonular dehiscence leading to vitreous loss or loss of sulcus support, was recorded.

\section{Statistical Analysis}

All statistical analyses were performed using Stata version 13 . Chi-squared analysis was performed for categorical data and analysis of variance was used for continuous variables. A generalized estimating equation model was constructed to determine factors (age, sex, race, complex case, laterality, surgical technique, and year) that were associated with the occurrence of intraoperative complications. For surgical technique, pop and chop was used as the reference variable against all other surgical methods. Statistical significance was set at $p<0.05$.

\section{Results}

A total of 1,253 resident cataract surgeries involving 988 patients were performed at our institute over the 5 -year study period. Mean age of the included participants was $64.7 \pm 14.05$ years (range, 5.8-98.1 years) and 57.6\% $(n=570 / 988)$ were females. Black patients made up $58.6 \%(n=579 / 988)$ and white patients made up $31.7 \%$ ( $n=313 / 988)$ of our sample. Complex cases accounted for $41.7 \%(n=522 / 1253)$ of the total patient population, ranging from $37.7 \%(n=101 / 268)$ at the beginning of the study to $44.3 \%(n=185 / 418)$ in the later academic years ( $\mathbf{- T a b l e ~} \mathbf{1}$ ).

With respect to preoperative risk factors, $16.7 \%$ ( $n=209 / 1253)$ patients had a dense cataract, $10.1 \%$ $(n=127 / 1253)$ had a small pupil, $8.1 \%(n=101 / 1253)$ had high myopia, $6.1 \%(n=76 / 1253)$ had history of ocular trauma, 5.5\% $(n=69 / 1253)$ had undergone prior vitrectomy, 3.5\% $(n=44 / 1253)$ had pre-existing zonular weakness, $3.0 \%$ $(n=37 / 1253)$ had pseudoexfoliation, and $1.0 \%(n=12 / 1253)$ had posterior polar cataracts.

We found statistically significant differences for the rates of capsular staining $(p<0.001)$, use of pupil expansion devices $(p<0.001)$, and the nuclear disassembly technique $(p<0.001)$ used across the academic years. Capsular staining decreased from $76.1 \%(n=204 / 268)$ in 2013 to 2014 to $63.4 \%$ $(n=130 / 205)$ in 2017 to 2018 , whereas the use of pupil expansion devices increased from $3.0 \%(n=8 / 268)$ in 2013 to 2014 to $9.8 \%(n=20 / 205)$ in 2017 to 2018 (-Table 2).

Overall, intraoperative complications occurred in 45/1253 (3.6\%) cases across the study period, with a trend toward a 
Table 1 Patient characteristics and demographics for resident cataract cases supervised by a single attending grouped by academic year

\begin{tabular}{|c|c|c|c|c|c|c|}
\hline & 2013-2014 & 2014-2015 & 2015-2016 & 2016-2017 & 2017-2018 & $p$-Value \\
\hline Subject level & $(n=210)$ & $(n=227)$ & $(n=226)$ & $(n=166)$ & $(n=159)$ & \\
\hline Age (y) & & & & & & $0.05^{a}$ \\
\hline Mean \pm SD & $62.8 \pm 15.4$ & $66.6 \pm 11.9$ & $63.3 \pm 13.9$ & $63.7 \pm 14.0$ & $64.8 \pm 16.3$ & \\
\hline Range & $10.8-93.5$ & $19.5-92.3$ & $7.7-89.2$ & $5.9-94.1$ & $9.0-98.1$ & \\
\hline Sex, $n(\%)$ & & & & & & $0.70^{b}$ \\
\hline Male & $87(41.4)$ & $90(39.6)$ & $99(43.8)$ & $77(46.4)$ & 65 (40.9) & \\
\hline Female & $123(58.6)$ & $137(60.4)$ & $127(56.2)$ & $89(53.6)$ & $94(59.1)$ & \\
\hline Race, $n(\%)$ & & & & & & $0.14^{\mathrm{b}}$ \\
\hline Black & $132(62.9)$ & $140(61.4)$ & $127(55.9)$ & $95(54.6)$ & $85(50.6)$ & \\
\hline White & $54(25.7)$ & $62(29.2)$ & $75(33.0)$ & $64(36.8)$ & $58(34.5)$ & \\
\hline Other & $24(11.4)$ & $26(11.4)$ & $25(11.0)$ & $15(8.6)$ & 25 (14.9) & \\
\hline Eye level & $(n=268)$ & $(n=295)$ & $(n=272)$ & $(n=213)$ & $(n=205)$ & \\
\hline Complex case $^{c}$ & $101(37.7)$ & $116(39.3)$ & $120(44.1)$ & $100(46.9)$ & 85 (41.5) & $0.23^{b}$ \\
\hline
\end{tabular}

Abbreviations: ANOVA, analysis of variance; SD, standard deviation.

${ }^{\text {a ANOVA. }}$

${ }^{\mathrm{b}}$ Chi-squared analysis.

'Complex case is defined as cases with one or more preoperative risk factor (small pupil, pre-existing zonular weakness, white or brunescent cataract, history of ocular trauma, pseudoexfoliation syndrome, myopia great than -6 diopters, prior history of vitrectomy).

Table 2 Intraoperative characteristics for resident cataract cases supervised by a single attending grouped by academic year

\begin{tabular}{|c|c|c|c|c|c|c|}
\hline & $\begin{array}{l}2013-2014 \\
(n=268)\end{array}$ & $\begin{array}{l}2014-2015 \\
(n=295)\end{array}$ & $\begin{array}{l}2015-2016 \\
(n=272)\end{array}$ & $\begin{array}{l}2016-2017 \\
(n=213)\end{array}$ & $\begin{array}{l}2017-2018 \\
(n=205)\end{array}$ & $p$-Value ${ }^{a}$ \\
\hline Capsular staining, $n(\%)$ & $204(76.1)$ & 205 (69.5) & $138(50.7)$ & $135(63.4)$ & $130(63.4)$ & $<0.001$ \\
\hline Expansion device, $n$ (\%) & $8(3.0)$ & $6(2.0)$ & $24(8.8)$ & $25(11.7)$ & $20(9.8)$ & $<0.001$ \\
\hline Nuclear disassembly technique, $n(\%)$ & & & & & & $<0.001$ \\
\hline Pop and chop & $55(20.5)$ & $29(9.8)$ & $106(39.0)$ & $89(41.8)$ & $47(23.6)$ & \\
\hline Bag-in-the-lens ${ }^{b}$ & $174(64.9)$ & $208(70.5)$ & $125(46.0)$ & $100(46.9)$ & $129(64.8)$ & \\
\hline Other $^{c}$ & $39(14.6)$ & $58(19.7)$ & $41(15.0)$ & $22(10.3)$ & $23(11.6)$ & \\
\hline
\end{tabular}

${ }^{a}$ Chi-squared analysis.

${ }^{\mathrm{b}}$ Divide and conquer, stop and chop, primary chop, horizontal.

'Small incision cataract surgery, irrigation/aspiration.

decreasing rate of intraoperative complications. The rate of complications decreased to $70 \%$, from $6.7 \%(n=18 / 268)$ in 2013 to 2014 to $2.0 \%(n=4 / 204)$ in 2017 to $2018(p=0.03)$ (-Table 3).

On multivariable analysis, we found an inverse relationship between intraoperative complications and experience of the supervising surgeon $(p=0.01)$, reflected by the decreasing rates of intraoperative complications with each successive year of supervision by the attending surgeon. Complications were also less likely during the end of the academic year than at the start $(p=0.03)$. We also found that complications during cataract surgery were more likely to occur for black patients when compared with white patients $(p=0.02)$, in eyes with preoperative risk factors $(p<0.001)$, and in left eyes compared with right eyes $(p=0.02)$. There were no associations of intraoperative

Table 3 Rate of intraoperative complications for resident cataract cases supervised by a single attending grouped by academic year

\begin{tabular}{|c|c|c|c|c|c|c|}
\hline & $\begin{array}{l}2013-2014 \\
(n=268)\end{array}$ & $\begin{array}{l}2014-2015 \\
(n=295)\end{array}$ & $\begin{array}{l}2015-2016 \\
(n=272)\end{array}$ & $\begin{array}{l}2016-2017 \\
(n=213)\end{array}$ & $\begin{array}{l}2017-2018 \\
(n=204)\end{array}$ & $p$-Value ${ }^{a}$ \\
\hline No complications, $n(\%)$ & $250(93.3)$ & $287(97.3)$ & $262(96.3)$ & $208(98.3)$ & $200(98.0)$ & \multirow[t]{2}{*}{0.030} \\
\hline Complications, $n$ (\%) & $18(6.7)$ & $8(2.7)$ & $10(3.7)$ & $5(1.9)$ & $4(2.0)$ & \\
\hline
\end{tabular}

${ }^{\mathrm{a} C h i-s q u a r e d}$ analysis. 
Table 4 Factors associated with occurrence of complications

\begin{tabular}{|l|l|l|}
\hline Variable & $\begin{array}{l}\text { Odds ratio } \\
\text { (95\% confidence } \\
\text { interval) }\end{array}$ & $p$-Value \\
\hline Age & $\begin{array}{l}0.98 \\
(0.97-1.01)\end{array}$ & 0.18 \\
\hline $\begin{array}{l}\text { Sex (reference female) } \\
\text { Male }\end{array}$ & $\begin{array}{l}1.53 \\
(0.79-2.95)\end{array}$ & 0.21 \\
\hline $\begin{array}{l}\text { Race (reference White) } \\
\text { Black }\end{array}$ & $\begin{array}{l}2.46 \\
(1.13-5.36)\end{array}$ & 0.02 \\
\hline $\begin{array}{l}\text { Risk factor } \\
\text { Present }\end{array}$ & $\begin{array}{l}4.79 \\
(2.35-9.76)\end{array}$ & $<0.001$ \\
\hline $\begin{array}{l}\text { Laterality } \\
\text { (reference right eye) } \\
\text { Left eye }\end{array}$ & $\begin{array}{l}2.18 \\
(1.13-4.19)\end{array}$ & 0.02 \\
\hline Year & $\begin{array}{l}0.72 \\
(0.56-0.92)\end{array}$ & 0.01 \\
\hline $\begin{array}{l}\text { Pop and chop } \\
\text { (reference no) }\end{array}$ & $\begin{array}{l}1.14 \\
(0.57-2.30)\end{array}$ & 0.71 \\
\hline $\begin{array}{l}\text { Yes } \\
\text { (reference July-October }{ }^{\mathrm{a}} \text { ) } \\
\text { November-February } \\
\text { March-June }\end{array}$ & $\begin{array}{l}\text { b } \\
(0.28-1.14)\end{array}$ & 0.03 \\
\hline
\end{tabular}

${ }^{a}$ Beginning of academic year.

${ }^{\mathrm{b}}$ End of academic year.

complications with age, sex, or surgical technique (-Table 4).

\section{Discussion}

In this retrospective analysis of over 1,200 cataract cases, we found an important association between the supervising surgeon's experience and the resident phacoemulsification learning curve, with a trend toward decreasing intraoperative complication rates with increasing experience of the supervising surgeon. In fact, the intraoperative complication rate decreased almost $25 \%$ with every passing year of supervision by the surgeon. Comparable findings were previously reported at our institute by Puri et al who reported an initial complication rate of $28 \%$ for the first 25 cases supervised by the novice attending surgeon compared with $6.67 \%$ in the last 15 cases over a 1-year study period. ${ }^{7}$ Between 2013 and 2018, PGY4 residents at our institute have been comparable in terms of the number of cataract surgeries performed prior to the cataract surgery rotation in their final year of training (typically $<10$ cases performed). Moreover, the wetlab has been part of the resident surgical training curriculum opened since 2013, thereby allowing for similar wetlab exposure between classes.

Therefore, we believe that these findings may in part be attributable to the growing development of the supervising surgeon as an educator that comes with experience. Literature has demonstrated that experience is crucial for surgical educators to develop their own skillset as a surgeon themselves. Campbell et al showed significant reductions in surgeon complication rates up until the tenth year of their own independent practice. ${ }^{10}$ Thus, with time, feedback, and drawing on their own experiences as a surgeon, a surgical educator can learn to refine their teaching methods and their delivery style to a manner that residents may find most effective. For example, in the operating room, seasoned surgical educators can draw upon past experiences making them potentially more adept at predicting intraoperative complications and intervening in a timely and efficient manner. Thus, recognizing the importance of the novice attending surgeon's learning curve is salient in ensuring patient safety and reducing surgical risk. We also found complications were less likely to occur toward the end of the academic year than the start of the academic year, a finding consistent with one of our earlier studies and we believe this may be related to the resident phacoemulsification learning curve. Residents may be less proficient at cataract surgery at the beginning of the academic year owing to fewer cataract cases performed at that point. Thus, it may be useful for supervising attendings to be particularly diligent during the first 4 months of the academic year to avoid potential complications.

Our finding of decreasing use of capsular staining with increased experience of the supervising surgeon is consistent with that reported by Puri et al. ${ }^{7}$ In their study, the authors concluded that while capsular staining has the potential to serve as a surrogate marker for case complexity, the novice attending surgeon may have employed the use of Trypan blue to ensure safe measures when overseeing residents during capsulorhexis formation. The increasing use of iris expansion devices in our cohort may have served a similar purpose to facilitate safe surgery during challenging cases. Moreover, our data corroborated existing literature on intraoperative complications-including the association of case complexity with an increased risk of intraoperative complications. ${ }^{11,12}$

Our observation of black patients being at increased odds of experiencing an intraoperative complication than their white counterparts may be related to racial disparities in utilization of eye care resources. The resident clinic at our institution primarily serves an underserved population in East Baltimore who are less likely to establish routine healthcare, ${ }^{13,14}$ potentially resulting in later presentation with more advanced cataracts. We found that although the number of dense cataracts ( $4+$ or more) was comparable between blacks and whites ( 16.4 vs. $17 \%, p=0.98$ ), the use of intraoperative capsular staining to be significantly higher for black patients compared with whites (68.6 vs. $56.5 \%$, $p<0.001$ ). No differences were, however, observed in the use of pupil expansion devices between the two racial groups (6.9 in blacks vs. $6.6 \%$ in whites, $p=0.83$ ). Interestingly, however, we found that the presence of preoperative risk factors was significantly higher among white patients (51.9\%) than blacks (36\%). We believe that these observations warrant further investigation into possible reasons for racial differences in cataract surgery outcomes. Finally, surgeons directly out of training and adapting to their new role of resident supervision should exercise particular caution for complex cases, which were 4.5 times more likely to experience an intraoperative complication in our study population compared with eyes that did not have any preoperative risk factors present. 
The role of the surgical educator cannot be underestimated, as it affects the training experience of residents as well as patient outcomes. Surgical educators are responsible for balancing patient safety, operating room efficiency, and resident learning needs while supervising trainees in the operating room. As such, teaching cataract surgery to residents can often be a stressful experience for the attending surgeon ${ }^{14}$ and much like learning the skill to perform surgery independently, teaching cataract surgery is a skill that comes with experience. Novice surgical educators, because of limited first operator experience, may, therefore, have higher levels of stress that can affect the quality of teaching and the ability to communicate effectively with the resident. However, no formal training currently exists for educating surgical educators to teach cataract surgery to trainees. A national survey of 950 UK-based ophthalmologists found that $83 \%$ of the respondents had received no formal training in how to teach cataract surgery. ${ }^{15}$ Given the diverse role surgical educators play in resident surgical education, residency programs may, therefore, consider organizing specific sessions and structured curriculums for educating the educators, with increased emphasis toward surgeons at the beginning of their teaching career. Furthermore, implementation of certain educational resources, including lectures and case-based discussions where faculty and learners can reflect on their experiences, collaborate on teaching and learning activities, support each other through successes and mistakes, and model reciprocal feedback should, be considered. Individual one-onone discussions with more experienced attending surgeons may also be valuable. These efforts will not only prepare attending surgeons for their role as surgical educators but also enhance the learning experience of residents. Further research should focus on the role of such educational resources in the development of skills for novice surgical educators.

Some limitations of our study include its retrospective design and that only cases performed by PGY4 residents were assessed. Since PGY4 residents are exposed to cataract surgery in their early training years, it would, therefore, be beneficial to assess cataract surgery outcomes for trainees during their initial cataract cases when supervised by novice surgeons.

Another possible limitation of our study is that we included cases that were supervised by only one attending surgeon at a single academic institute, which may limit the generalizability of our study. Future studies examining the complication rates associated with novice attending surgeons involved in resident teaching across multiple ophthalmology residency programs are warranted.

\section{Conclusions}

In summary, we found that learning curves may exist for novice attending surgeons that can also influence outcomes of resident-performed cataract surgery. Novice surgical educators should exercise caution early in their career, particularly while supervising challenging cases with underlying preoperative risk factors. Residency programs should aim to create top surgical educators by promoting a teaching culture. Future work is needed to determine which educational resources are most useful to surgical educators and whether a structured curriculum for educators can reduce surgical complications.

Funding

None.

\section{Conflict of Interest}

None declared.

\section{Acknowledgments}

The authors would like to acknowledge the Wilmer Biostatistical department (Core Grant EY01765) for assistance with data analysis.

\section{References}

1 Randleman JB, Wolfe JD, Woodward M, Lynn MJ, Cherwek DH, Srivastava SK. The resident surgeon phacoemulsification learning curve. Arch Ophthalmol 2007;125(09):1215-1219

2 Taravella MJ, Davidson R, Erlanger M, Guiton G, Gregory D. Characterizing the learning curve in phacoemulsification. J Cataract Refract Surg 2011;37(06):1069-1075

3 Borboli-Gerogiannis S, Jeng-Miller KW, Koulisis N, et al. A comprehensive surgical curriculum reduced intra-operative complication rates of resident-performed cataract surgeries. J Surg Educ 2019;76(01):150-157

4 Rogers GM, Oetting TA, Lee AG, et al. Impact of a structured surgical curriculum on ophthalmic resident cataract surgery complication rates. J Cataract Refract Surg 2009;35(11):1956-1960

5 Subramonian K, Muir G. The 'learning curve' in surgery: what is it, how do we measure it and can we influence it? BJU Int 2004;93 (09):1173-1174

6 Maruthappu M, Duclos A, Lipsitz SR, Orgill D, Carty MJ. Surgical learning curves and operative efficiency: a cross-specialty observational study. BMJ Open 2015;5(03):e006679

7 Puri S, Kiely AE, Wang J, Woodfield AS, Ramanathan S, Sikder S. Comparing resident cataract surgery outcomes under novice versus experienced attending supervision. Clin Ophthalmol 2015;9:1675-1681

8 Mahmoudi E, Lu Y, Chang S-C, et al. The associations of hospital volume, surgeon volume, and surgeon experience with complications and 30-day rehospitalization after free tissue transfer: a national population study. Plast Reconstr Surg 2017;140(02):403-411

9 Corey RP, Olson RJ. Surgical outcomes of cataract extractions performed by residents using phacoemulsification. J Cataract Refract Surg 1998;24(01):66-72

10 Campbell RJ, El-Defrawy SR, Gill SS, et al. New surgeon outcomes and the effectiveness of surgical training: a population-based cohort study. Ophthalmology 2017;124(04):532-538

11 Blomquist PH, Morales ME, Tong L, Ahn C. Risk factors for vitreous complications in resident-performed phacoemulsification surgery. J Cataract Refract Surg 2012;38(02):208-214

12 Zare M, Javadi M-A, Einollahi B, Baradaran-Rafii A-R, Feizi S, Kiavash V. Risk factors for posterior capsule rupture and vitreous loss during phacoemulsification. J Ophthalmic Vis Res 2009;4(04):208-212

13 Tielsch JM, Sommer A, Katz J, Quigley H, Ezrine S; Baltimore Eye Survey Research Group. Socioeconomic status and visual impairment among urban Americans. Arch Ophthalmol 1991;109(05):637-641

14 Zhang X, Cotch MF, Ryskulova A, et al. Vision health disparities in the United States by race/ethnicity, education, and economic status: findings from two nationally representative surveys. Am J Ophthalmol 2012;154(6, Suppl):S53-62.e1

15 Alexander P, Matheson D, Baxter J, Tint NL. United Kingdom national cataract training survey. J Cataract Refract Surg 2012; 38(03):533-538 